\section{Sistemas de informação em saúde e monitoramento de morbidade materna grave e mortalidade materna}

\section{Health information systems and surveillance of severe maternal morbidity and maternal mortality}

\author{
Maria Helena de Sousa 1 \\ José Guilherme Cecatti 2 \\ Ellen Elizabeth Hardy 3 \\ Eliana Amaral 4 \\ João Paulo Dias de Souza 5 \\ Suzanne Serruya 6
}

\begin{abstract}
1-5 Departamento de Tocoginecologia. Faculdade de Ciências Médicas. Universidade Estadual de Campinas. Rua Alexander Fleming, 101. Campinas, SP, Brasil. CEP: 13.083.881 E-mail: cecatti@unicamp.br

6 Departamento de Ciência, Tecnologia e Insumos Estratégicos.
\end{abstract} Ministério da Saúde. Brasília, DF, Brasil.

\begin{abstract}
During the last decades there was a huge decrease of maternal mortality in developed countries, while in developing countries the decrease was small or did not happen at all. Nevertheless, because maternal death is less common in absolute frequency, severe maternal morbid events became the focus of attention because these are "lookout events" preceding death. In Brazil, the main source for health information is the Ministry of Health, through its system called DATASUS. The use of this available routine information by several public health information systems could be useful not only to determine severe maternal morbidity and mortality, but also for prospective and continuous monitoring. This could potentially create a surveillance system allowing, when activated, the implementation of preventive and/or therapeutic interventions to avoid maternal death. It could be an innovative and challenging method to be tested in a near future.
\end{abstract}

Key words Morbidity, Maternal mortality, Information systems

\section{Resumo}

Nas últimas décadas, nos países desenvolvidos houve queda acentuada da mortalidade materna, enquanto nos países em desenvolvimento a queda foi pequena ou não ocorreu. De qualquer forma, como o óbito materno, em termos absolutos, é pouco freqüente, os eventos mórbidos maternos graves tornaram-se o foco das atenções, por representarem "eventos sentinela" ao óbito. No Brasil a principal fonte de informações em saúde é o Ministério da Saúde, através de seu sistema chamado DATASUS. A utilização dessas informações rotineiras, disponíveis nos diversos sistemas de informação públicos de saúde pode ser útil não apenas para o mapeamento diagnóstico de morbidade grave e mortalidade materna, mas também para seu monitoramento contínuo e prospectivo, criando um sistema de alerta ou de vigilância que permita, quando ativado, desencadear intervenções terapêuticas ou preventivas para evitar a ocorrência do óbito materno, constituindo uma inovação e um desafio a ser testado em futuro próximo.

Palavras-chave Morbidade, Mortalidade materna, Sistemas de informação 


\section{Mortalidade materna}

A morte de uma mulher no período da gravidez, no parto ou no puerpério é um evento que finaliza, de forma trágica, o processo natural da reprodução humana. A definição de morte materna, segundo a Organização Mundial da Saúde (OMS), ${ }^{1}$ é a de uma mulher durante a gestação ou dentro de um período de 42 dias após o término da gestação, independente da duração ou da localização da gravidez, devido a qualquer causa relacionada com ou agravada pela gravidez ou por medidas em relação a ela, porém não devido a causas acidentais ou incidentais.

Em países desenvolvidos, como os Estados Unidos e países do Reino Unido, a razão de mortalidade materna foi alta até a década de 30 , ao redor de 650/100.000 e 400/100.000 nascidos vivos, respectivamente. Um declínio acentuado foi verificado nos anos seguintes, atingindo, em 1960, menos de 50 mortes por 100.000 nascidos vivos. 2 Tal fato não aconteceu em países em desenvolvimento, de tal forma que a quase totalidade das mortes maternas vem ocorrendo justamente nesses países.3,4

A World Health Organization (WHO), em conjunto com o United Nations International Children's Emergency Fund (UNICEF), estimou para o ano de 1990 um total de 585.000 e para 2000 cerca de 529.000 mortes maternas no mundo, sendo 99\% dessas em países do chamado "Terceiro Mundo" 5,6 o que sugere a morte materna como um indicador sensível das desigualdades sociais mundialmente existentes. Destaca-se que não há outro indicador de saúde pública que mostre maior disparidade entre os países, em função de sua riqueza e desenvolvimento, que a mortalidade materna. ${ }^{7}$

Contudo, ainda segundo Loudon, ${ }^{2}$ as diferenças na mortalidade materna dos países desenvolvidos, nas primeiras décadas do século XX, não se deveram unicamente a mudanças sociais e econômicas, mas principalmente foram relacionadas aos cuidados médicos e obstétricos oferecidos. Como exemplo, em um estudo realizado na década de 70, Crawford e Opit 8 apontaram as necessidades quanto aos cuidados anestésicos em unidades obstétricas de uma região da Inglaterra, com o objetivo de também reduzir as mortes maternas indiretas decorrentes de causas relacionadas à anestesia.

Em 1987, na primeira Conferência Internacional sobre Maternidade Segura, em Nairobi, no Quênia, estabeleceu-se a meta de redução de $50 \%$ nos níveis de mortalidade materna até o ano 2000.3 Conferências internacionais posteriores voltaram a apoiar essa meta. Entretanto, poucos países em desenvolvimento têm mostrado redução importante nos níveis de mortalidade, como Sri Lanka, China, Cuba e Malásia. Esses exemplos reforçam a suposição de que a condição econômica de um país pode não ser o determinante mais importante da ocorrência das mortes. ${ }^{9}$

$\mathrm{Na}$ virada para o século XXI, constatou-se que pouca ou nenhuma melhora havia ocorrido nas taxas de mortalidade materna na maioria dos países em desenvolvimento, atestando o insucesso do compromisso assumido pelos países no "Programa de Maternidade Segura". 9 Embora tenha se mostrado a tendência de uma lenta redução em algumas partes do mundo, a mortalidade materna continua extremamente elevada em regiões menos favorecidas econômica e socialmente, como em vários países africanos. ${ }^{10}$ A possível explicação para o fracasso em se obter a redução de $50 \%$ no período de pouco mais de 10 anos, é que mudanças eficazes na assistência à saúde são difíceis de serem implementadas através de programas e, mais difíceis ainda são mudanças socioeconômicas em nível populacional. Além disso, esse período pode ser curto para se avaliar o impacto de programas sobre a ocorrência da mortalidade materna.

Existe uma aceitação universal de que as principais causas desencadeantes do óbito materno podem ser classificadas como: obstétricas diretas, quando resultantes de complicações exclusivas à gestação; obstétricas indiretas, quando resultantes de condições pré-existentes, mas agravadas pela gestação; e não obstétricas ou não relacionadas, quando resultantes de outras causas acidentais ou incidentais que aconteceram durante a gravidez, mas sem relação com ela. Em termos gerais, as causas diretas de morte materna correspondem a aproximadamente $80 \%$ do total de eventos e, portanto, são consideradas como passíveis de serem evitadas. ${ }^{11}$ As principais causas diretas são a hemorragia, com aproximadamente $25 \%$ do total de casos, seguida pela infecção com $15 \%$, as complicações de aborto inseguro com $13 \%$ e a eclâmpsia com $12 \% .9$ Essas proporções variam de país a país e entre regiões, com uma tendência de diminuição de causas hemorrágicas e aumento de causas por hipertensão à medida que aumenta o desenvolvimento.

Devido ao insucesso na redução substancial da mortalidade materna em países pobres de uma maneira geral, e pelo fato da maioria dos óbitos pertencer ao grupo de causas obstétricas diretas, têm sido avaliados mais detalhadamente os programas nacionais e/ou regionais que visam tal redução. Analisam-se ainda outros indicadores que melhor e mais facilmente expressem as complicações associadas à gravidez, parto e puerpério e que potencial- 
mente levem ao óbito materno.

A dificuldade em identificar fatores predisponentes associados à ocorrência dos óbitos maternos pode estar relacionada ao fracasso desses programas, porque, em números absolutos, os óbitos são poucos. A partir do momento em que a morte materna tornase um acontecimento raro, os estudos sobre suas causas começam a tratar, cada vez mais, de ocorrências muito incomuns, cuja frequiência é praticamente irrelevante para a assistência obstétrica da maioria das mulheres, pois mesmo análises detalhadas de poucos óbitos não possibilitariam propor mudança de conduta na rotina do cuidado obstétrico. ${ }^{12}$ Além do mais, quando se investigam os fatores relacionados a um óbito, percebe-se claramente a dificuldade relativa à falta da principal fonte de informação, ou seja, o depoimento do próprio indivíduo, especialmente quando fatores sociais e de funcionamento do sistema estão mais freqüentemente envolvidos.

\section{Morbidade materna grave}

Em países desenvolvidos, onde as razões de mortalidade materna são baixas, em geral inferiores a 20/100.000 ou 10/100.000 nascidos vivos, a preocupação com a adequada assistência persiste, uma vez que muitas mulheres ainda sofrem com sérios agravos à sua saúde associados à gravidez. $\mathrm{O}$ estudo dos eventos mórbidos graves que colocam em risco a vida das mulheres passa a constituir uma nova categoria de análise, com as mulheres sobreviventes. Além dessa motivação, as condições de morbidade materna grave associadas à gravidez, parto e puerpério podem representar "eventos sentinela" ao óbito materno, permitindo melhor estudar e identificar as situações predisponentes para o mesmo. O estabelecimento dessa identificação de modo prospectivo e sistemático permitiria trazer evidentes benefícios à saúde materna.

O termo near miss surgiu e ainda hoje é utilizado em controle de tráfego aéreo para descrever um possível acidente, quando dois aviões se aproximam o suficiente para justificar uma preocupação ou alarme, ainda que o acidente mesmo não ocorra de fato. Esse termo é um conceito útil que pode ser aplicado em várias situações clínicas. ${ }^{13} \mathrm{Na}$ área da saúde materna, surgiu no final da década de 80 , fazendo referência a um evento adverso sério que tenha levado a um dano e morbidade para a mãe, mas que não causou a morte, e tem sido cada vez mais explorado até os dias de hoje.13-18

Mantel et al.,14 em 1998, apresentaram uma proposta piloto, em termos conceituais, descrevendo como near miss as mulheres que sofreram importantes agravos sistêmicos, que se não tratadas apropriadamente poderiam evoluir para óbito. Um conjunto de sinais sentinelas foi estabelecido para a definição de casos. Além de alguns dados clínicos específicos, inclui todas as pacientes admitidas nas Unidades de Terapia Intensiva (UTI), as submetidas a histerectomia de emergência e as que sofreram acidentes anestésicos. Com uma revisão retrospectiva, Stones et al., 16 em 1991, investigaram o padrão de morbidade obstétrica e a freqüência de episódios sérios ameaçadores da vida da mulher, ampliando a análise da assistência com maior representatividade.

Assim, na última década, diversos estudos foram realizados para estudar pacientes obstétricas internadas em Unidade de Terapia Intensiva, considerando tais pacientes como casos de near miss, ou seja, de morbidade materna grave.19-23 A descrição das condições patológicas apresentadas por essas pacientes tem apontado algumas causas principais, que são as doenças hipertensivas e hemorragias, além das infecções e suas complicações.20,21,23-27 Com isso, há autores que apóiam o estudo das pacientes obstétricas admitidas em UTI, como refletindo um grupo próximo àquele das mortes maternas. Embora esse critério seja discutível, uma vez que é fortemente influenciado por questões da organização da assistência e pode apresentar uma ampla variação na definição das admissões, alguns estudos adotam especificamente a transferência de pacientes para as unidades de maior porte de complexidade, como casos de near miss, considerando que somente quadros de grave morbidade obstétrica necessitam de serviços de grande complexidade.

Alguns pesquisadores têm questionado, ainda, se é suficiente estudar apenas as pacientes internadas em UTI como sendo aquelas que estão sob risco de morte materna. 14,28 Questionam que muitas mulheres com problemas obstétricos graves podem não chegar a ser internadas em UTI, enquanto outras que de fato o foram, podem não estar em risco iminente de morte, sobretudo em locais com melhores recursos, onde a disponibilidade dessas unidades é maior.

Como conceito recente na literatura médica, near miss é ainda uma categoria nova, cujo desenho metodológico não pode ser tão objetivo quanto o usado nos estudos de mortalidade materna, existindo, portanto, uma discussão sobre a definição de casos entre os autores, nos diferentes estudos. Prual et al.29 definiram itens relacionados a causas obstétricas diretas. A problemática da definição de casos de near miss e sua utilidade em novas audito- 
rias de acidentes obstétricos foram discutidas por Drife ${ }^{30}$ que sugere a avaliação desses casos separadamente dos inquéritos confidenciais de morte materna. Waterstone et al. ${ }^{31}$ selecionaram definições que fossem clinicamente baseadas e rotineiramente medidas, sem incluir processos de manejo, e colocam a possibilidade de discussão e modificação dessa definição. Dessa forma, não há, até o momento, um consenso final sobre a definição de near miss, e alguns autores destacam a necessidade de se ter uma definição precisa, para que comparações possam ser feitas. 13,32-35

Em recente revisão sobre o tema, Minkauskiene et al. 12 resumiram os achados de 24 estudos, a maioria deles $(67 \%)$ de base hospitalar. Concluíram que a prevalência de morbidade materna grave variou de $0,7 / 1.000$ a 82,3/1.000 NV, com diferentes métodos de avaliação utilizados. Eles destacam a necessidade de melhorar a qualidade dos registros de morbidade, além de se desenvolver uma clara definição dos casos de near miss. Os múltiplos critérios utilizados pelos autores que estudam o assunto incluem a hemorragia obstétrica, doença hipertensiva e suas complicações, embolia pulmonar, complicações anestésicas, rotura uterina, necessidade de UTI, de transfusão de sangue e hemoderivados, alterações cardíacas ou pulmonares agudas, hepáticas, renais, do sistema nervoso central, da coagulação, metabólicas, infecção, procedimentos complementares como curetagem, histerectomia e laparotomia, e necessidade de ventilação mecânica, entre outros.

Os resultados de alguns estudos 14,17,20,29,31,34 permitem uma comparação e apontam para um conjunto de condições patológicas já relatadas do ponto de vista epidemiológico como prevalentes, além de identificarem em que casos os tratamentos estiveram incorretos ou fora do padrão necessário. Nos estudos em que as sobreviventes são entrevistadas, também é possível identificar quais fatores contribuem para a situação vivida, do ponto de vista da usuária e de sua comunidade. Tudo isso leva ao argumento de que talvez possam de fato variar os critérios para a caracterização do near miss em diferentes contextos.

Estes dados apontam para taxas mais altas de near miss em países em desenvolvimento, tal qual na mortalidade materna. Algumas questões que são determinantes para a mortalidade, também são muito relevantes para a morbidade grave, como o acesso adequado a uma assistência pré-natal e ao parto, além de suportes tecnológicos específicos, como a presença de unidade de terapia intensiva, ou mesmo o acesso a transfusões com a necessária rapidez. Nos casos de atenção a mulheres com morbidade obstétrica severa, os serviços e recursos humanos envolvidos necessitam, além de maior organização e disponibilidade de recursos, inicialmente reconhecer tais quadros clínicos e saber como conduzi-los.

O padrão de morbidade obstétrica encontrado aponta para o mesmo conjunto de causas reconhecidas em países de alta mortalidade materna. Hipertensão, quadros hemorrágicos e infecções aparecem, com diferentes percentuais, como as primeiras causas de morte. Isso permitiria identificar um conjunto significativo de mulheres cuja assistência demandaria especialistas capacitados, recursos mais complexos e maior disponibilidade de serviços, como laboratório e banco de sangue.

Todo o conhecimento adquirido nas últimas décadas sobre mortalidade materna e morbidade materna grave como um possível indicador de óbito, tem apontado no sentido de que intervenções dirigidas especificamente ou somente à redução dos óbitos maternos não têm atingido seu objetivo a curto prazo, pelo menos em países em desenvolvimento. Além disso, que a utilização dos conceitos ampliados de near miss parece ter uma boa relação com morte materna, com a vantagem da maior facilidade de estudar "mulheres vivas" do que "mulheres mortas", somado ainda à perspectiva de implementação de medidas terapêuticas e/ou preventivas para impedir a morte.

$\mathrm{Na}$ Escócia, 36 foi sugerido o estabelecimento de um sistema nacional para o relato de morbidade materna, assim como ocorre para os casos de óbito. Essas medidas representam iniciativas operacionais onde o conceito poderia ser aplicado na prática como um sistema de vigilância epidemiológica. Para essa situação, entretanto, há necessidade de um sistema local completamente desenvolvido que permita, não só a identificação prospectiva de tais situações, como também a ativação sistemática de condutas derivadas de protocolos padronizados, com o objetivo de evitar as possíveis mortes.

\section{Mortalidade e morbidade materna grave no Brasil}

Apesar da tendência de queda da mortalidade materna, que no período de 1980 a 1994 teve redução de $35 \%, 37$ esse indicador continua alto no Brasil. Segundo estimativa da Pesquisa Nacional sobre Demografia e Saúde (PNDS) de 1996, a razão de mortalidade materna (RMM) para o Brasil foi estimada em 160 por 100.000 nascidos vivos, 38 embora os dados oficiais não corrigidos mostrem 
uma RMM de 51,6 óbitos por 100.000 nascidos vivos em 199739 e 64,8 óbitos por 100.000 nascidos vivos em 1998.40

A redução da mortalidade materna não é tarefa simples, devido aos múltiplos fatores envolvidos. Entretanto, é conveniente salientar a importância dos Comitês de Morte Materna que, considerando-a um evento em geral evitável, muito podem contribuir com a discussão dos casos fatais. ${ }^{41}$ Além disso, iniciativas governamentais têm sido muito úteis no contexto da saúde materna, como a publicação de textos técnicos sobre o assunto, como o recente guia abordando ações a serem tomadas em situações de urgências e emergências maternas, 39 bem como o estabelecimento de um pacto nacional pela redução da mortalidade materna, prevendo reduzir em $15 \%$ a RMM nas capitais brasileiras, no período 2004 a 2007.42

Em relação a assistência à mulher, deve ser ofertado planejamento familiar a todas as mulheres em idade reprodutiva, para redução de gravidez indesejada e possível aborto. Além disso, medidas como a assistência de qualidade no pré-natal, parto e puerpério, devem ser oferecidas a todas as gestantes. 39 Também, esforços podem ser feitos no sentido de se detectar precocemente eventos que podem colocar em risco a vida da mulher. Esse conjunto de recomendações já faz parte dos diversos programas de saúde coordenados nacionalmente, contando, inclusive, com guias técnicos de condutas, desenvolvidos exatamente com este propósito. Mesmo assim, as dificuldades para a real implementação dessas medidas provavelmente estão associadas com restrições quantitativas e qualitativas dos serviços em lograrem êxito na prevenção e controle da morbidade materna. Uma análise dos principais indicadores da saúde materna mostra que, apesar do incremento no número de consultas no pré-natal e do melhor desempenho dos outros indicadores, não houve impacto positivo na razão de morte materna. Para a mudança desse quadro é necessário articulação entre os diferentes níveis da atenção, com indispensável hierarquização, além da implementação de medidas já largamente recomendadas por todos os programas de saúde materna. 43

Assim como ocorre no mundo, as causas diretas de morte materna também se constituem na maioria dos eventos no Brasil e, portanto, são passíveis de serem evitadas em sua quase totalidade. ${ }^{44,45}$ Quanto aos estudos publicados envolvendo o conceito de near miss materna no Brasil, são apenas três e restritos a internações em UTI.21,27,46-48 O primeiro deles relata uma incidência de 8,2/1.000 partos, com as doenças hipertensivas e hemorrágicas correspon- dendo a mais da metade dos casos, 21 enquanto o segundo obteve uma prevalência de 2,4/1.000 partos, com as principais indicações para transferência à UTI as desordens hipertensivas (41\%), hemorragia (15\%) e sepse (13\%).27 O fato de ambos os estudos usarem apenas uma definição reduzida, referente às pacientes admitidas/transferidas à UTI, pode explicar esses valores não tão elevados da razão de near miss. O terceiro estudo procura explorar os diferentes conceitos de near miss e sua utilização para o diagnóstico das condições de morbidade materna grave em uma maternidade de referência. $46-48$ De forma semelhante ao que acontece em todo o mundo, não existem ainda estudos nacionais que explorem o conceito de near miss em nível populacional.

\section{Informações sobre mortalidade e morbidade materna}

Não apenas para o Brasil, mas também para outros países de uma forma geral, seria desejável dispor de um sistema de informações, a partir de dados rotineiramente coletados, que permitisse monitorar a morbidade materna grave, implantando medidas específicas para seu controle individual ou coletivo, a fim de evitar a ocorrência do óbito, ou seja, um verdadeiro sistema de vigilância epidemiológica. Esse seria um grande desafio do sistema público com o objetivo de melhorar a saúde reprodutiva das mulheres, e que demandaria vontade política de priorizar essa meta.

Até onde se tem conhecimento, nenhum país possui em vigor um sistema regular de vigilância epidemiológica em morbidade materna que utilize rotineiramente dados hospitalares e de registro vital. Entretanto, recentemente estão surgindo iniciativas em alguns países, como Canadá, Estados Unidos e Honduras, para o monitoramento da morbidade materna grave através desses sistemas de informação. ${ }^{49-51}$ Um estudo do Sistema de Vigilância Perinatal Canadense, considerando a base de dados de hospitalizações e de registros vitais, bem como a participação dos comitês regionais de morte materna, sugeriu ser esse um primeiro passo para auditoria também em morbidade materna, além da mor-talidade. ${ }^{49}$ Nos Estados Unidos tem sido enfatizada a utilização de dados nacionais rotineiros de alta hospitalar. 50 Até mesmo em país em desenvolvimento, como é o caso de Honduras, o tópico de utilização de informações hospitalares vem sendo destacado, inclusive com o desenvolvimento de um sistema piloto de vigilância em saúde materna.51 
No Brasil, a principal fonte de informações em saúde é o Ministério da Saúde, através do Departamento de Informática do Sistema Único de Saúde (DATASUS). Pela rede mundial de computadores (Internet), dados dos Sistemas de Informações sobre Mortalidade (SIM), sobre Nascidos Vivos (SINASC) e sobre Informações Hospitalares (SIH/SUS), entre outros, estão disponíveis às pessoas interessadas que podem obter tabelas específicas de uma maneira relativamente fácil. As informações do SIM estão disponíveis desde o ano de 1979 , as de nascidos vivos desde 1991, e as informações hospitalares mensais, a partir de 1994.52 Tais dados são de domínio público, porém poucos pesquisadores os utilizam de forma contínua e sistemática. A proposta de utilização desses sistemas de informação do SUS, para avaliar a possibilidade de propor um monitoramento da saúde das mulheres em idade reprodutiva, através dos óbitos e das ocorrências de morbidade materna grave, poderá modificar a abordagem do diagnóstico da situação e a maneira como manejá-la.

Assim como na maioria dos países em desenvolvimento, no Brasil os registros de saúde possuem confiabilidade questionável. Com isso, mesmo existindo, não há uma garantia da qualidade total da informação. Entretanto, a estrutura do sistema brasileiro de coleta e armazenamento de informações em saúde é considerada como razoavelmente bem elaborada. Caso a informação seja correta e adequadamente inserida no sistema e depois também adequadamente analisada e interpretada, pode se constituir em importante ferramenta para os mais diferentes propósitos de diagnóstico, mudança de políticas e implementação de sistemas de vigilância em saúde.

Alguns autores brasileiros têm apresentado métodos que permitem integrar dados rotineiramente coletados nos diversos bancos disponíveis. Como exemplo, Campos et al. ${ }^{53}$ apresentaram um procedimento operacional para combinar os diversos arquivos parciais que compõem o SIH, obtendo-se um único banco de dados com informações dos pacientes, do processo de cuidado e dos profissionais, em conjunto. Outros estudos, que estão recentemente sendo desenvolvidos em nosso contexto, tratam da aplicação do método de relacionamento probabilístico de registros, que busca combinar os vários sistemas existentes, como o SIM e o SIH, quando não existe um campo único e comum de identificação nos mesmos. 54,55

Em função da maioria dos estudos brasileiros em near miss ser institucional e, portanto, de limitada validade para a generalização populacional, o que se propõe para estudos futuros é a tentativa de se utilizar os dados de rotina do DATASUS para, além de buscar uma definição de morbidade materna grave, avaliar a viabilidade de se utilizar tal definição como um indicador de predição da mortalidade materna, para um monitoramento contínuo desses eventos críticos.

Os sistemas informatizados do SUS possuem diversas informações complementares para a obtenção de indicadores em saúde materna. O SIH, apesar de ter sido concebido para fins administrativos, apresenta informações de diagnósticos e procedimentos das internações que poderiam ser utilizados para obter dados de morbidade. Já o SIM possui a causa básica do óbito, e o SINASC, informações sobre os nascidos vivos. Com isso, tais dados podem ser teoricamente utilizados para se obter a razão de mortalidade materna utilizando o SIM e o SINASC, e a razão de morbidade materna grave utilizando o SIH e o SINASC, isso pressupondo uma boa qualidade da informação existente nesses bancos.

Em se tratando de morte materna, evento já ocorrido, a importância seria a de se disponibilizar uma estimativa bastante atualizada de sua ocorrência, com a possibilidade de ser mapeada em função das regiões e estados. No caso específico da morbidade materna, e mais especialmente da morbidade materna grave, o near miss, a importância é ainda maior, porque conceitualmente estaria em jogo a possibilidade de se disponibilizar, a tempo, intervenções ou medidas terapêuticas dirigidas especificamente para o suporte e o controle das complicações da gravidez, parto e/ou puerpério. De fato, se o sistema hospitalar permitisse um diagnóstico precoce e uma intervenção adequada, na própria instituição ou em outra para onde a mulher pudesse ser referenciada, estaria então caracterizado um verdadeiro sistema de vigilância, através do qual tornaria possível intervir na cadeia de acontecimentos de morbidade que pode acabar resultando na morte materna. Esse parece ser de fato um desafio, que teria um impacto significativo sobre a qualidade da saúde das mulheres durante o processo reprodutivo, e que poderia desempenhar uma nova linha de ação e investimento, sobretudo dos países em desenvolvimento, para o combate das situações associadas à ocorrência das mortes maternas.

\section{Considerações finais}

Em países desenvolvidos, onde a mortalidade materna apresenta números absolutos abaixo de 10/100.000 NV, a proposição de análise dos casos de morbidade materna grave justifica-se como uma 
nova medida da qualidade dos cuidados obstétricos, buscando reduzir o evento trágico que é o óbito da mulher em idade reprodutiva, mas fundamentalmente desvendando o panorama de processos patológicos responsáveis pela morbidade materna.

Em países em desenvolvimento, como o Brasil, a mesma proposta pode ser feita com o intuito de se aprofundar e antecipar a análise de casos que, sem um correto diagnóstico e tratamento, podem evoluir ao óbito. As inúmeras ações que buscam a diminuição da mortalidade materna nesses países têm tido pouco impacto, em médio prazo. Como predominam as causas obstétricas diretas, é de vital importância a assistência em todas as fases, ou seja, do pré-natal ao puerpério, para que ações efetivas sejam tomadas.

Idealmente, assim como ocorre em países desenvolvidos, os países em desenvolvimento deveriam ter condições de diagnosticar precocemente e tratar as urgências e emergências maternas, garantindo, com o menor risco possível, o processo natural da

\section{Referências}

1. OMS (Organização Mundial da Saúde). Classificação Estatística Internacional de Doenças e Problemas Relacionados à Saúde: 10. revisão. 8. ed. São Paulo: Centro Colaborador da OMS para a Classificação de Doenças em Português, Ed. Universidade de São Paulo; 2000. v.1.

2. Loudon I. The transformation of maternal mortality. BMJ. 1992; 305: 1557-60.

3. Mahler H. The safe motherhood initiative: a call to action. Lancet. 1987; 1: 668-70.

4. Barns T. Obstetric mortality and its causes in developing countries. Br J Obstet Gynaecol. 1991; 98: 345-8.

5. WHO (World Health Organization), UNICEF (United Nations International Children's Emergency Fund). Revised 1990 estimates of maternal mortality. Geneva; 1996.

6. Abouzahr C, Wardlaw T. Maternal mortality in 2000: estimates developed by WHO, UNICEF and UNFPA. Geneva; 2003.

7. Duley L. Maternal mortality associated with hypertensive disorders of pregnancy in Africa, Asia, Latin America and the Caribbean. Br J Obstet Gynaecol. 1992; 99: 547-53.

8. Crawford JS, Opit LJ. A survey of the anaesthetic services to obstetrics in the Birmingham Hospital Region: a summary. Anaesthesia. 1976; 31: 56-9.

9. WHO (World Health Organization). Reduction of maternal mortality: a joint WHO/UNFPA/UNICEF World Bank Statement. Geneva; 1999.

10. Abouzahr C, Wardlaw T. Maternal mortality at the end of a decade: signs of progress? Bul World Health Organ. 2001; 79: $561-73$.

11. Global Forum for Health Research. The 10/90 report on health research 2001-2002. Geneva; 2002. reprodução humana. A utilização de informações rotineiras em saúde, disponíveis no território nacional nos diversos sistemas de informação públicos de saúde (SIM, SINASC e SIH, entre outros), pode ser útil não apenas para o mapeamento diagnóstico de morbidade grave e mortalidade materna, mas também para seu monitoramento contínuo e prospectivo, criando um sistema de alerta ou de vigilância que permita, quando ativado, desencadear intervenções terapêuticas ou preventivas para a ocorrência do óbito materno, constituindo uma inovação e um desafio a ser testado em futuro próximo. O objetivo deste artigo foi, assim, fazer uma revisão sobre o tema em discussão, focalizando especificamente a promissora possibilidade de utilização de estatísticas oficiais disponíveis nos sistemas de informação sobre dados hospitalares (SIH), numa tentativa de diagnóstico precoce de situações mórbidas graves associadas à gravidez que pudessem indicar um possível óbito materno com a evolução. Esse é o desafio que nos propomos a enfrentar.

12. Minkauskiene M, Nadisauskiene R, Padaiga Z, Makari S. Systematic review on the incidence and prevalence of severe maternal morbidity. Medicina. (Kaunas) 2004; 40 : 299-309.

13. Nashef SAM. What is a near miss? Lancet. 2003; 361: 1801.

14. Mantel GD, Buchmann E, Rees H, Pattinson RC. Severe acute maternal morbidity: a pilot study of a definition for a near-miss. Br J Obstet Gynaecol. 1998; 105: 985-90.

15. Graham SG, Luxton C. The requirement for intensive care support for the pregnant population. Anaesthesia. 1989; 44: 581-4.

16. Stones W, Lim W, Al-Azzawi F, Kelly M. An investigation of maternal morbidity with identification of life-threatening 'near miss' episodes. Health Trends. 1991; 23: 13-5.

17. Filippi V, Ronsmans C, Gandaho T, Graham W, Alihonou E, Santos P. Women's reports of severe (near miss) obstetric complications in Benin. Stud Fam Plann. 2000; 31: 309-24.

18. Khosla AH, Dahiva K, Sangwan K. Maternal mortality and "near miss" in rural north India. Int J Gynaecol Obstet. 2000; 68: 163-4.

19. Fitzpatrick C, Halligan A, McKenna P, Coughlan BM, Darling MR, Phelan D. Near miss maternal mortality (NMM) [letter]. Ir Med J. 1992; 85: 37.

20. Baskett TF, Sternadel J. Maternal intensive care and nearmiss mortality in obstetrics. Br J Obstet Gynaecol. 1998; 105: 981-4.

21. Viggiano MGC, Viggiano MB. A necessidade de cuidados intensivos e o termo "near-miss mortality" aplicado à realidade obstétrica brasileira. Ginecol Obstet Atual. 2000; 9: 29-32. 
22. Ni Bhuinneain M, Barry-Kinsela C, Coughlan BM McKenna P, Bosio P. Critical care admission of obstetric patients [letter]. Ir Med J. 2001; 94: 26.

23. Murphy DJ, Charlett P. Cohort study of near-miss maternal mortality and subsequent reproductive outcome. Eur J Obstet Gynecol Reprod Biol. 2002; 102: 173-8.

24. Bouvier-Colle MH, Salanave B, Ancel PY, Varnoux N, Fernandez H, Papiernik E, Breart G, Benhamou D, Boutroy P, Caillier I, Dumoulin M, Fournet P, Elhassani M, Puech F, Poutot C. Obstetric patients treated in intensive care units and maternal mortality. Regional teams for the survey. Eur J Obstet Gynecol Reprod Biol. 1996; 65: 121-5.

25. Mahutte NG, Murphy-Kaulbeck L, Le Q, Solomon J, Benjamin A, Boyd ME. Obstetric admissions to the intensive care unit. Obstet Gynecol. 1999; 94: 263-6.

26. Tripathi R, Rathore AM, Saran S. Intensive care for critically ill obstetric patients [brief communication]. Int $\mathrm{J}$ Gynaecol Obstet. 2000; 68: 257-8.

27. Dias de Souza JP, Duarte G, Basile Filho A. Near-miss maternal mortality in developing countries [letter]. Eur J Obstet Gynecol Reprod Biol. 2002; 104: 80.

28. Bouvier-Colle MH. Maternal intensive care and near-miss mortality in obstetrics [letter]. Br J Obstet Gynaecol. 1999; 106: 1234

29. Prual A, Bouvier-Colle MH, De Bernis L, Bréart G. Severe maternal morbidity from direct obstetric causes in West Africa: incidence and case fatality rates. Bull World Health Organ. 2000; 78: 593-602.

30. Drife JO. Maternal "near miss" reports? BMJ. 1993; 307: 1087-8.

31. Waterstone M, Bewley S, Wolfe C. Incidence and predictors of severe obstetric morbidity: case-control study. BMJ. 2001; 322: 1089-93.

32. Penny J. Severe acute maternal morbidity: a pilot study of a definition of a near-miss [letter]. Br J Obstet Gynaecol. 1999; 106: 397.

33. Paruk F, Moodley J. Severe obstetric morbidity. Curr Opin Obstet Gynecol. 2001; 13: 563-8.

34. Sahel A, Brouwere VD, Lardi M, Lerberghe WV, Ronsmans C, Filippi V. Des catastrophes obstétricales évitées de justesse: les near miss dans les hôpitaux Marocains. Santé. 2001; 11: 229-35

35. Geller SE, Rosenberg D, Cox SM, Kilpatrick S. Defining a conceptual framework for near-miss maternal morbidity. J Am Med Women's Assoc. 2002; 57: 135-9.

36. Brace V, Penney G, Hall M. Quantifying severe maternal morbidity: a Scottish population study. $\mathrm{Br}$ J Obstet Gynaecol. 2004; 111: 481-4.

37. Levine R, Glassman A, Schneidman M. La salud de la mujer en América Latina y el Caribe. Washington (DC): Banco Interamericano de Desarrollo; 2001. p. 19-20.

38. BEMFAM (Sociedade Civil Bem-Estar Familiar no Brasil), Macro International. Pesquisa Nacional sobre Demografia e Saúde: 1996. Rio de Janeiro; 1997.

Recebido em 23 de agosto de 2004

Versão final apresentada em 14 de fevereiro de 2006

Aprovado em 25 de fevereiro de 2006
39. Ministério da Saúde. Secretaria de Políticas de Saúde. Área Técnica da Saúde da Mulher. Guia para diagnóstico e conduta em situações de risco de morte materna. Brasília (DF): 2000

40. Ministério da Saúde. Secretaria de Políticas de Saúde. Área Técnica de Saúde da Mulher Manual dos comitês de mortalidade materna. 2. ed. Brasília (DF); 2002.

41. Faúndes A, Cecatti JG, organizadores. Morte materna: uma tragédia evitável. 2. ed. Campinas: Ed. UNICAMP; 1991.

42. Ministério da Saúde. Secretaria de Atenção à Saúde. Departamento de Ações Programáticas Estratégicas. Política Nacional de Atenção Integral à Saúde da Mulher: plano de ação 2004-2007. Brasília (DF); 2004.

43. Serruya SJ, Lago TG, Cecatti JG. O panorama da atenção pré-natal no Brasil e o Programa de Humanização do PréNatal e Nascimento. Rev Bras Saúde Matern Infant. 2004; 4: $269-79$.

44. Cecatti JG, Albuquerque RM, Hardy E, Faúndes A. Mortalidade materna em Recife: causas de óbitos maternos. Rev Bras Ginec Obstet. 1998; 20: 7-11.

45. Cecatti JG, Faúndes A, Surita FGC. Maternal mortality in Campinas: evolution, under-registration and avoidance. São Paulo Med J. 1999; 117: 5-12.

46. Souza JPD, Cecatti JG, Parpinelli MA. Fatores associados à gravidade da morbidade materna na caracterização do near miss. Rev Bras Ginecol Obstet. 2005; 27: 197-203.

47. Dias de Souza JP, Cecatti JG. The near-miss maternal morbidity scoring system was tested in a clinical setting in Brazil [letter]. J Clin Epidemiol. 2005; 58: 962.

48. Dias de Souza JP, Cecatti JG, Parpinelli MA, Sousa MH, Serruya SJ. Revisão sistemática sobre morbidade materna near miss. Cad Saúde Pública. 2006; 22: 255-64.

49. Canada. Minister of Public Works and Government Services. Health Canada: special report on maternal mortality and severe morbidity in Canada - enhanced surveillance: the path to prevention. Ottawa; 2004.

50. Berg CJ, Bruce FC, Callaghan WM. From mortality to morbidity: the challenge of the twenty-first century. J Am Med Womens Assoc 2002; 57: 173-4.

51. USAID (United States Agency for International Development). Developing and implementing a hospitalbased surveillance system for maternal and newborn health. Disponível em <http://www.mnh.jhpiego.org/global/ dvlsrvsys.asp $>$. [2005 May 15].

52. FNS (Fundação Nacional de Saúde), Sistema Único de Saúde (SUS). Departamento de Informática (DATASUS). Diretório de bases de dados. Disponível em: <http://datasus.gov. br/dirbd/estrut.htm>. [2003 abr 4].

53. Campos MR, Martins M, Noronha JC, Travassos C. Proposta de integração de dados do Sistema de Informações Hospitalares do Sistema Único de Saúde (SIH-SUS) para pesquisa. Inf Epidemiol SUS 2000; 9: 51-8.

54. Camargo Jr KR, Coeli CM. Reclink: aplicativo para o relacionamento de bases de dados, implementando o método probabilistic record linkage. Cad Saúde Publica. 2000; 16 : 439-47.

55. Coeli CM, Camargo Jr KR. Avaliação de diferentes estratégias de blocagem no relacionamento probabilístico de registros. Rev Bras Epidemiol. 2002; 5: 185-96. 